\title{
LEVELS OF AFLATOXINS AND FUMONISINS IN POULTRY FEED FROM GHANA
}

\author{
Justice KUMI ${ }^{\circledR 凶}$, Kofi Aaron A-O AGYEI-HENAKU² and Mark OFOSUHENE ${ }^{1}$ \\ ${ }^{1}$ Noguchi Memorial Institute for Medical Research, University of Ghana, Legon P.O Box LG 581, Accra-Ghana \\ ${ }^{2}$ Central University, Agribusiness Management Department, P.O.Box TM 2305, Tema-Ghana \\ Email: jkumi@noguchi.ug.edu.gh; ORCID: 0000-0002-5929-2322 \\ supporting Information
}

\begin{abstract}
Mycotoxins are secondary fungal metabolites that contaminate animal feeds, crops and food. Globally, two major foodborne mycotoxins (aflatoxins and fumonisins) have been reported to affect the health and productivity of the poultry industry. Notwithstanding the health risks associated with these mycotoxins, no study has probably investigated the co-occurrence of aflatoxin and fumonisin levels in poultry feed produced in Ghana. The aim of the study was to investigate the levels of total aflatoxin (B1, B2, and G1 and G2) and fumonisin $B 1\left(\mathrm{FB}_{1}\right)$ levels in poultry feed produced in Ghana. Total aflatoxins and fumonisin $B 1$ were analyzed in 100 poultry feed samples collected from farmers from four major poultry producing regions in Ghana. High performance liquid chromatography (HPLC) was used to measure total aflatoxin levels whiles a fluorometer reader was used to measure the levels of fumonisin $\mathrm{B} 1$. Total aflatoxin and $\mathrm{FB}_{1}$ contaminations were detected in $100 \%$ of the feed samples in a range of $0.02-22 \mathrm{ppb}$ and $0.5-4.6 \mathrm{ppm}$, respectively. Three samples representing, $3 \%$ out of the 100 samples screened were detected to have total aflatoxin levels greater than 20 parts per billion Fumonisin levels detected in the poultry feed were within the permissible level of 50 parts per million in poultry feed. Seventy out of the 100 poultry feed samples collected had their feed treated with fungal binders. In present study, we have probably for the first time in Ghana shown the levels of mycotoxins in poultry feed and the need to monitor animal feed made from cereals.
\end{abstract}

Keywords: Poultry feed, Total aflatoxin, Fumonisin B1, Ghana

\section{INTRODUCTION}

Mycotoxins (aflatoxin and fumonisin) are toxic secondary metabolites produced by certain fungi in agricultural products that are susceptible to mould infestation. Mycotoxin contamination is difficult to predict, which makes it a challenge to food safety (Park and Stoloff, 1989). Aflatoxin (AFB 1 being the most toxic) is produced on various food crops including maize, beans and groundnuts by Aspergillus flavus and Aspergillus parasiticus. Fumonisin $\mathrm{B}_{1}$ being the most toxic, is produced primarily in maize by Fusarium verticulliodes and Fusarium monoliforme (Martins et al., 2001; Voss and Haschek, 2007). Environmental conditions such as high temperature, humidity, poor soil fertility, drought, insect damage, as well as the food production chains are characteristics in most parts of Africa where diets primarily consist of maize which is susceptible to fungal growth and mycotoxins production (Lewis et al., 2005).

Contamination of food products by mycotoxins can take place at any point along the food/feed chain from the field, harvest, handling, shipment and storage under a wide range of climatic conditions (Giray et al., 2007). Aflatoxin poisoning is one of the most common and most under-reported causes of toxicoses in poultry (Pattison et al., 2007). Aflatoxicosis in poultry and farm animals also causes changes in biochemical and hematological parameters, liver and kidney abnormalities, impaired immunity, and may induce mortality among animals, secondary contamination of human consumers via eggs, meat and milk (Shashidhara and Devegowda, 2003). Fumonisin $\left(\mathrm{FB}_{1}\right)$ also suppresses the immune system, causes deficiency in nutrients such as folic acid (Blom et al., 2006) and the modification of sphingomyelin metabolism (Stockmann-Juvala and Savolainen, 2008).

Research conducted in Nigeria, Tanzania and India on animal feed commodities showed mycotoxin contamination (Kehinde et al., 2014). Kajuna et al. (2013) in a study involving poultry feed in Tanzania reported that, $68 \%$ of all feed samples were contaminated with aflatoxin $B_{1}$. In Haryana, India, poultry feed samples analysed by Jindal et al. (1999) contained fumonisin $B_{1}$ contamination.

According to FAO report (2013), the rate of population growth over the past five decades has increased globally and has led to the rapid growth in the meat sector by rising demand of poultry meat, which has increased 
by threefold. The poultry industry has been a contributing area in agriculture that leads the economy with $34.5 \%$ share of the total growth domestic product in Ghana (Attuahene et al., 2012). Poisoning of poultry feed with mycotoxin could affect the total growth per domestic product of economy growth if not well investigated. Globally, maize (corn) and soybean are used in the preparation of poultry feed (Ensminger et al., 1990). Studies in Ghana have demonstrated contamination of weanimix food (a cereal-legume blend food) with aflatoxin and fumonisin (Kumi et al., 2014). Since a large number of poultry feed are prepared from these cereals, there is the possibility of mycotoxins contamination of poultry feed from Ghana. However, studies regarding aflatoxin and fumonisin levels in poultry feed in the animal industry are readily not available in Ghana.

Thus, the aim of the study was to investigate the levels of total aflatoxin (B1, B2, G1 and G2) and fumonisin B1 in poultry feed produced in Ghana.

\section{MATERIALS AND METHODS}

\section{Study sites}

Four major poultry producing regions in Ghana, which include Brong Ahafo, Ashanti, Accra and Western regions of Ghana, were selected for this study. The Brong Ahafo region is the largest poultry producing region which contributes to about $29.62 \%$ of poultry production followed by, $28.07 \%, 10.72 \%$ and $9.99 \%$ from Ashanti, Eastern and Western regions of Ghana respectively (FAO, 2014).

\section{Sample collection}

The poultry feed samples, which were made up of a blend of maize, wheat grain and wheat bran were collected from farmers who produced their own feed locally in Ghana. One hundred poultry farms were selected randomly for the study. Forty samples were collected from Accra, 20 from Ashanti, 21 from Western and 19 from Brong Ahafo regions of Ghana. Hundred grams of poultry feed samples, which were made up of layer mash, broiler starter, and grower mash were collected into sterile zip-locked bags and transported at room temperature to the Noguchi Memorial Institute for Medical Research. All laboratory work was conducted at the Noguchi Memorial Institute for Medical Research, University of Ghana.

\section{Aflatoxin measurement}

Sample preparation. About $50 \mathrm{~g}$ of poultry feed was measured and blended with $5 \mathrm{~g}$ sodium chloride $(\mathrm{NaCl})$ and $100 \mathrm{mls}$ of $\mathbf{8 0} \%$ methanol in a blender at high speed for 1-2 minutes. The supernatant was collected and filtered twice using a filter paper. The filtered supernatant $(10 \mathrm{~mL})$ was measured into a clean vessel, diluted with $40 \mathrm{~mL}$ of distilled water. Diluted extract was filtered twice through glass microfibre filter into a clean vessel.

Affinity chromatography. The diluted extract, $5 \mathrm{~mL}$ was passed through Afla Test affinity column at a rate of about 1- 2 drops/second. The column was washed with $10 \mathrm{~mL}$ of distilled water. The extracted aflatoxin in the poultry feed was eluted with1.0 mL HPLC grade methanol into a clean test tube.

Total aflatoxin quantification. Total aflatoxin quantification was done using a calibrated high-performance liquid chromatography with fluorescence detection of wavelength $365 \mathrm{~nm}$ excitation and 425nm emission.

\section{Fumonisin measurement}

Sample extraction. Poultry feed sample $(50 \mathrm{~g})$ and $5 \mathrm{~g}$ sodium chloride $(\mathrm{NaCl})$ were measured and blended with $100 \mathrm{~mL}$ of $80 \%$ methanol in a blender for $1-2$ minutes. The supernatant was collected and filtered twice using a filter paper and diluted 4-fold with $0.1 \%$ Tween-20.

Affinity chromatography. Five $(5 \mathrm{~mL})$ of filtered diluted extract was passed through fumonitest affinity column at a rate of about 1- 2 drops/second followed by $10 \mathrm{~mL}$ wash with distilled water. Fumonisin in the feed was eluted by passing $1.0 \mathrm{~mL}$ HPLC grade of methanol through column at a rate of 1-2 drops/second while collecting the entire sample eluted in a test tube.

Fumonisin quantification. Fumonisin developer $(1.0 \mathrm{~mL})$ was added to the eluted sample, mixed and read at a fluorescence detection of $\mathbf{4 8 3 n m}$ after 240 seconds using a calibrated fluorometer (VICAM, series 4) with detection limit $0.2 \mathrm{ppm}(2 \mathrm{mg} / \mathrm{kg})$.

\section{Statistical analyses}

All statistical analyses were performed using Sigma Stat (2012). The data obtained from measurement of mycotoxins (total aflatoxin and fumonisin B1) from the four regions of Ghana (Accra, Ashanti, Western and Brong Ahafo) were represented by tables and figures and subjected to simple descriptive statistics, which looked at the range and the mean. 


\section{RESULTS}

Total aflatoxin (B1, B2, G1and G2) and fumonisin $B_{1}$ standard calibration curves were established with an $\mathrm{R}^{2}=$ 0.9913 and 0.9887 respectively (Figures 1 and 2) to determine the linearity of the HPLC system. Out of 100 samples collected, total aflatoxin contamination were detected in all samples in a range of 0.02-22 ppb. Three samples representing $3 \%$ of the total samples collected were detected to have total aflatoxin levels greater than 20 ppb (Table 1). Fumonisin contamination was detected in all 100 samples in the range of 0.5-4.6 ppm (Table 2). There was no statistical significant differences in occurrence of mycotoxins (total aflatoxin and fumonisin B1) from the various regions with $P=0.530$ and 0.612 for aflatoxin and fumonisin respectively ( $p$ significant was set to $<0.05$ with 95 percent confident level). Out of the 100 questionnaire analyses, 70 of the poultry feed samples collected, representing Greater Accra Region, Ashanti Region, Western Region, and Brong Ahafo Region had their feed mixed with antifungal binder to prevent fungal contamination whiles $30 \%$ did not use antifungal binders (Figure 3 ).

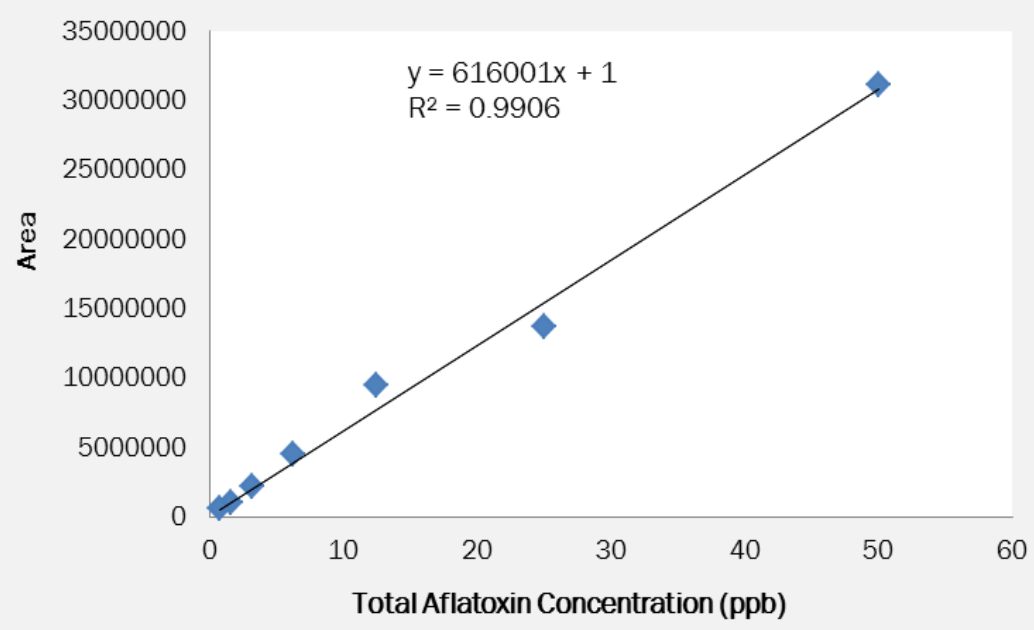

Figure 1 - Standard curve of total Aflatoxin, HPLC at excitation: 365nm, emission: 425nm

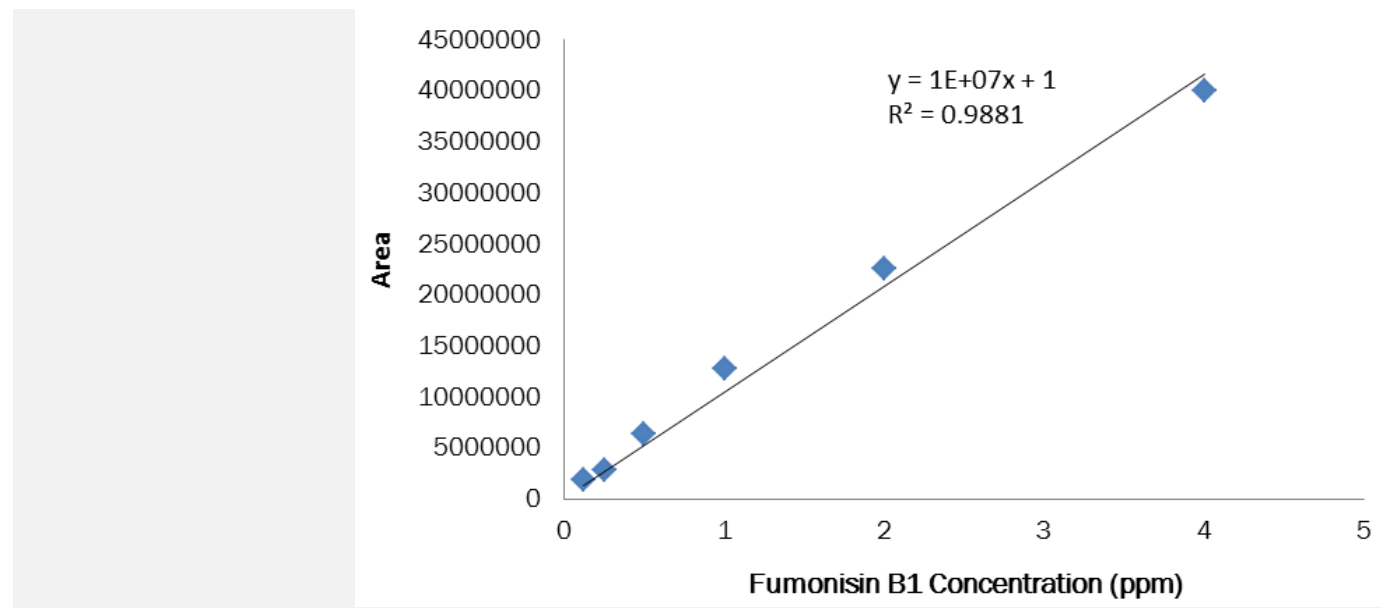

Figure 2 - Standard curve of Fumonisin $B_{1}$, at fluorescence $483 \mathrm{~nm}$

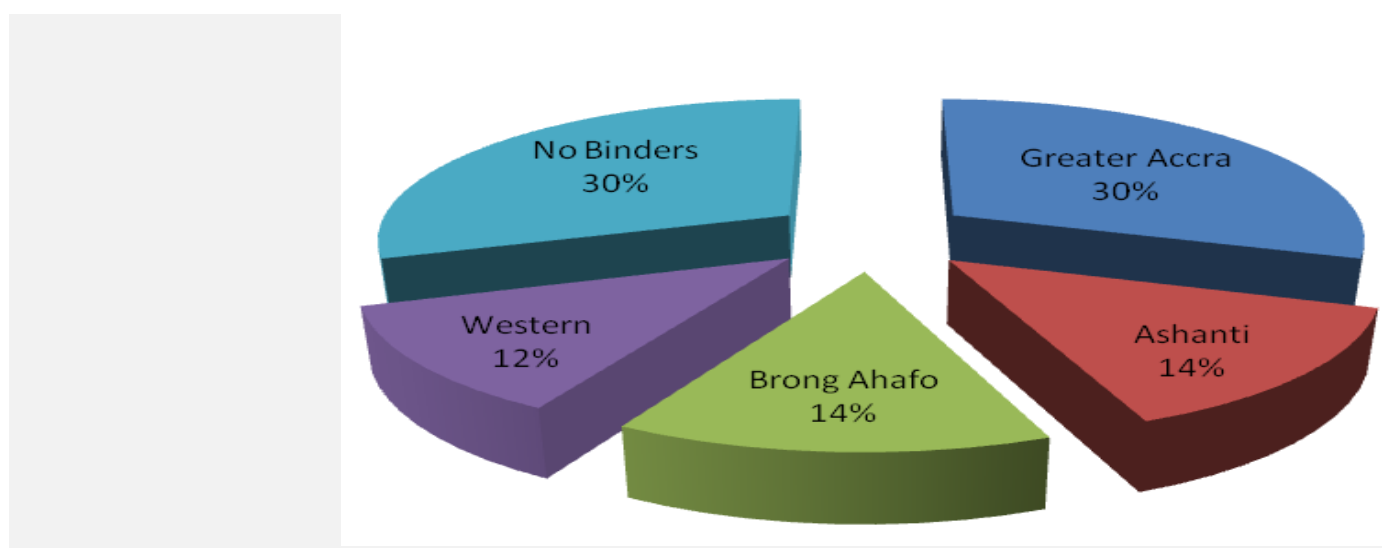


Table 1 - Total Aflatoxin Levels in Poultry feed Produced in Ghana

\begin{tabular}{lcccc} 
Region & $\begin{array}{c}\text { No of } \\
\text { Samples }\end{array}$ & $\begin{array}{c}\text { Mean TAF } \\
(\mathbf{p p b})\end{array}$ & $\begin{array}{c}\text { Range TAF } \\
(\mathbf{p p b})\end{array}$ & $\begin{array}{c}\text { TAF } \\
\mathbf{2}\end{array}$ \\
\hline Accra & 40 & 3.0 & $0.1-21$ & 1 \\
Ashanti & 20 & 2.4 & $0.05-22$ & 0 \\
Western & 21 & 1.3 & $0.02-3.9$ & 0 \\
Brong Ahafo & 19 & 4.7 & $0.8-22$ & 2 \\
n=100. TAF: Total Aflatoxin & & & \\
\hline
\end{tabular}

\section{Table 2 - Fumonisin B1 Levels in Poultry Feed Produced in Ghana}

\begin{tabular}{lcccc} 
Region & $\begin{array}{c}\text { Total No. of } \\
\text { Samples }\end{array}$ & $\begin{array}{c}\text { Mean FB } \\
(\mathbf{p p m})\end{array}$ & $\begin{array}{c}\text { Range FB } 1 \\
(\mathbf{p p m})\end{array}$ & $\begin{array}{c}\text { No Samples } \\
>50 \text { ppm }\end{array}$ \\
\hline Accra & 40 & 2.7 & $0.8-3.1$ & 0 \\
Ashanti & 20 & 1.5 & $0.3-4.6$ & 0 \\
Western & 21 & 1.2 & $0.08-1.4$ & 0 \\
Brong Ahafo & 19 & 0.3 & $0.5-1.5$ & 0 \\
n=100. FB: Fumonsin B1 & & & &
\end{tabular}

\section{DISCUSSION}

The poultry industry has been a contributing area in agriculture which leads the economy with $34.5 \%$ share of the total growth domestic product (GDP) in Ghana (Attuahene et al., 2012). Occurrence of mycotoxins in poultry feed and feed ingredients are of worldwide concern since they reduce poultry performance and could be important vehicles for introducing aflatoxins residues into the human diet (Ardic et al., 2008). Consequently, many countries have regulated the maximum permissible levels of aflatoxin in food and feed products to reduce the hazard of aflatoxin poisoning but in terms of its economic considerations; these regulations vary in different countries (Stoloff et al., 1991).

Mycotoxins (total aflatoxin and fumonisin B1) were detected in all poultry feed samples analysed. The United States Food and Drug Administration, regulatory affairs and center for veterinary medicine guidelines for aflatoxin regulate the limit of aflatoxin levels in poultry feed as $20 \mu \mathrm{g} / \mathrm{kg}$ (US FDA, 2019). According to the results of the present study, 97 feed samples representing $97 \%$ were below the permissible aflatoxin level of $20 \mathrm{ug} / \mathrm{kg}$ in poultry feed. Three feed samples representing $3 \%$ recorded total aflatoxin levels above the permissible level of (Table 1). The three feed samples which were above the permissible aflatoxin levels were from samples, received from farmers who did not add fungal binders to their feed (Figure 3). Similar to the findings of the present study, another study conducted in Iran reported low aflatoxin levels ranged between 0.05 and $5.38 \mu \mathrm{g} / \mathrm{kg}$ detected in 75 Iranian poultry feed samples (Mayahi et al., 2007). Aflatoxin B1 contamination was also found in sorghum and maize from India in the range 5-125 and 0.38-109 $\mathrm{mg} / \mathrm{kg}$ respectively which constitute the major ingredients of poultry feed (Shetty and Bhatl, 1997).

The presence of mycotoxin in poultry feed could results from the feed ingredients and raw materials used in their production (Lozada, 1995). Several mycotoxin binders have been developed that prevent harmful effects of mycotoxins on animals consuming contaminated feed. In a study conducted by Kolosova and Stroka (2012), commercial aflatoxin binders containing hydrated sodium calcium aluminosilicate was used as the main component in animal feed and showed a significant effect of $41 \%$ reduction on the amount of aflatoxin analysed. Seventy percent of the poultry feed obtained from farmers in the present study included fungal binders to their feed (Figure 3). The low levels of aflatoxin recorded in the present study could be due to the addition of fungal binders to poultry feed.

Maize and maize-based products were found to have the highest occurrence and mean concentrations of fumonisin B1 than any other cereal or cereal-based product in an evaluation by the Joint FAO/WHO Expert Committee meeting on Food Additives in 2016 in Rome, higher mean concentrations of fumonisin B1 was reported in products from Africa, Central and South America and some countries in the Western Pacific Region. In a study by Shetty and Bhat (1997), fumonisin B1 contamination in poultry feed were determined in the range of 0.02 to $0.26 \mathrm{mg} / \mathrm{kg}$. The present study reported fumonisin contamination in poultry feed in the range of $0.8-4.6 \mathrm{mg} / \mathrm{kg}$ (Table 2) which was lower than the permissible limit of $50 \mathrm{mg} / \mathrm{kg}$ set by the United States Food and Drug Administration (US FDA, 2001). The present study concurs to the report by Shetty and Bhat (1997) who reported similar findings. The low levels of fumonisin in poultry feed detected in the present study could be due to the fact 
that, majority of farmers who treated their feed with fungal binders (Figure 3). Majority of the poultry feed samples analysed had low levels of total aflatoxin and fumonisin B1.

CONCLUSION AND RECOMMENDATIONS

In accordance with the findings of the present study, it can be concluded that, most of the poultry feed samples collected and analyzed were within the permissible limit of total aflatoxin and fumonisin B1 consumption by poultry birds. Fungal binders were found to be helpful in reducing the amount of aflatoxin and fumonisin levels in poultry feed. Poultry farmers are encouraged to include antifungal binders to their feed before consumption by poultry birds. The present study for the first time in Ghana, probably has demonstrate the levels and the need to monitor poultry feed for mycotoxin contamination. However not withstanding farmers need to be educated on the dangers of mycotoxin poisoning to their birds.

\section{DECLARATIONS}

\section{Corresponding Author}

Justice Kumi, e-mail: jkumi@noguchi.ug.edu.gh

\section{Acknowledgement}

The authors are grateful to Central University, Accra-Ghana for providing sponsorship for the project and also to Lorreta Kwasah and Anita Houston Adams for helping in the proof reading.

\section{Authors' Contribution}

J.Kumi participated in the proposal design of the study, prepared the manuscript in writing and performed most of the laboratory analysis. Mr. Kofi Aaron Agyei-Henaku worked on reviewing and editing the proposal, recruitment of farmers into the study as well as contributed to laboratory analysis. Mark Ofosuhene contributed to the final proposal design. All authors have read the manuscript before submitting for publication.

\section{Availability of data and materials}

Data will be made available on request from the primary author.

Consent to publish

Not applicable.

Competing interests

The authors declare that they have no competing interests.

\section{REFERENCES}

Ardic M, Karakaya Y, Atasever M and Durmaz H (2008). Determination of aflatoxin B1 levels in deep- red ground pepper (isot) using immunoaffinity column combined with ELISA. Food and Chemical Toxicology, 46: 1596-1599. DOI: https://dx.doi.org/10.1016/j.fct.2007.12.025.

Attuahene CC, Atoh-Kotoku V and Mensah JJ (2012). Poultry production in Ghana, Prospects and Challenges. Ghana Journal of Animal Science. Vol. 5, No 2. http://ir.knust.edu.gh/xmlui/handle/123456789/6405

Blom HJ, Shaw GM, Heijer M, Finnell RH (2006). Neural tube defects and folate: case far from closed. Natures Reviews. Neuroscience, 7(9): 724-731. https://doi.org/10.1038/nrn1986

Ensminger ME, Oldfield JE and Heinemann WW (1990). Feeds and Nutrition. Second Edition, Ensminger Publishing Company, California, USA. https://www.worldcat.org/title/feeds-nutrition-formerly-feeds-nutrition-complete/oclc/20776611

Food and Agriculture Organisation (2014) Poultry Sector Ghana. FAO Animal Production and Health Livestock Country Reviews. No. 6. Rome.

Food and Agriculture Organization of the United Nations, Statistic Division (2013) Trends in the livestock sector, part 3, http://www.fao.org/docrep/015/i2490e/i2490e03c.pdf

Giray B, Girgin G, Engin AB, Aydin S and Sahin G (2007). Aflatoxin levels in wheat samples consumed in some regions of Turkey. Food Control, 18: 23-29. DOI: https://dx.doi.org/10.1016/j.foodcont.2005.08.002.

Joint FAO/WHO Expert Committee Meeting on Food Additives, 8-17 November (2016). Summary and reports of the eighty-third meeting Rome, http://www.fao.org/3/a-bq821e.pdf

Jindal N, Mahipal SK, Rottinghaus GE (1999). Occurrence of fumonisin $B_{1}$ in maize and poultry feeds in Haryana, India. Mycopathologia 148 (1): 37-40. PMID: 11086483 DOI: https://dx.doi.org/10.1023/a:1007162113368

Kajuna FF, Temba BA and Mosha RD (2013). Surveillance of aflatoxin B1 contamination in chicken commercial feeds in Morogoro, Tanzania. Livestock Research for Rural Development, 25, No 51. http://www.Irrd.org/Irrd25/3/kaju25051.htm

Kehinde MT, Oluwafemi F, Itoandon EE, Orji FA, Ajayi OI (2014). Fungal profile and aflatoxin contamination in poultry feeds sold in Abeokuta, Ogun State, Nigeria Food Journal. 32: 73-79. DOI: https://dx.doi.org/10.1016/S0189-7241(15)30098-9. 
Kolosova A and Stroka J (2012). Evaluation of the effect of mycotoxin binders in animal feed on the analytical performance of standardised methods for the determination of mycotoxins in feed. Food Additives and Contaminants Part A, 29: $1959-1971$. https://doi.org/10.1080/19440049.2012.720035

Kumi J, Mitchell N, Asare G, Dotse E, Kwaa F and Phillips T (2014). Aflatoxins and fumonisins contamination of home made food [Weanimix] from cereal-legume blends for children. Ghana Medical Journal, $48(3)$ : $121-126$ https://www.ncbi.nIm.nih.gov/pmc/articles/PMC4335443/

Lewis L, Onsongo M, Njapau H, Schurz-Rogers H, Luber G, Kieszak S, and Kenya Aflatoxicosis Investigation Group (2005). Aflatoxin contamination of commercial maize products during an outbreak of acute aflatoxicosis in eastern and central Kenya. Environmental Health Perspectives; 113(12): 1763-1767. DOI: https://doi.org/10.1289/ehp.7998

Lozada AF (1995). Isolation and identification of mycotoxigenic fungi in selected foods and feeds, Food Additives \& Contaminants, 12(3): 509-514, DOI: https://doi.org/10.1080/02652039509374337

Martins ML, Martins HM and Bernardo F (2001). Aflatoxins in spices marked in Portugal. Food Additives and Contaminants, 18 (4): 315-319. https://doi.org/10.1080/02652030120041

Mayahi M, Razi JM and Salamat N (2007). Isolation of Aspergillus spp and determination of aflatoxin level in fish meal, maize and soya meal. Shahid Chamran University Journal of Science, 17: 95-105.

Pattison M, McMullin P, Bradbury J and Alexander D (2007). Poultry Diseases (Sixth Edition), Chapter 38: 435-442. https://www.eu.elsevierhealth.com/vst-poultry-diseases-e-book-vst-9780702037269.html

Park DL and Stoloff L (1989). Aflatoxin control - how a regulatory agency managed risk from an unavoidable natural toxicant in food and feed. Regulatory Toxicology and Pharmacology Journal, 9: 109.

Shetty PH and Bhat RV (1997). Natural Occurrence of Fumonisin B1 and Its Co-occurrence with Aflatoxin B1 In Indian Sorghum, Maize, and Poultry Feeds. Journal of Agricultural and Food Chemistry, 45(6): 2170-2173. DOI: https://doi.org/10.1021/jf960607s

Shashidhara RG and Devegowda G (2003). Effect of dietary mannan oligosaccharide on broiler breeder production traits and immunity. Poultry Science, 82, 8: 1319-1325. DOI: https://doi.org/10.1093/ps/82.8.1319

Stoloff L, Park DL and Van Egmond HP (1991). Rationales for the establishments of limits and regulations for mycotoxins. Food Additive and Contaminants, 8: 213-221. DOI: https://doi.org/10.1080/02652039109373971

Stockmann-Juvala $\mathrm{H}$ and Savolainen K (2008). A review of the toxic effects and mechanisms of action of fumonisin B1. Hum Exp Toxicol, 27: 799-809. DOI: https://dx.doi.org/10.1177/0960327108099525.

United States Food and Drugs Authority. Guidance for Industry: Fumonisin Levels in Human Foods and Animal Feeds Revised November 9, 2001. https://www.fda.gov/regulatory-information/search-fda-guidance-documents/guidance-industryfumonisin-levels-human-foods-and-animal-feeds

United States Department of Health and Human, Services Food and Drug Administration, Office of Regulatory Affairs and Center for Veterinary Medicine Sec. 683.100. (2019). Action Levels for Aflatoxins in Animal Food Compliance Policy Guide. https://www.fda.gov/media/121202

Voss KA and Haschek WM (2007). Fumonisins: toxicokinetics, mechanism of action and toxicity. Science direct, animal feed science technology 137: 299-325. DOI: https://dx.doi.org/10.1016/j.anifeedsci.2007.06.007 